\title{
Differences In Male Collegiate and Recreationally Trained Soccer Players on Balance, Agility, and Vertical Jump Performance
}

\author{
Nicole M. Sauls, Nicole C. Dabbs* \\ Department of Kinesiology, California State University, San Bernardino, 5500 University Parkway, San Bernardino, CA 92407 \\ Corresponding Author: Nicole C. Dabbs, E-mail: ndabbs@csusb.edu
}

\section{ARTICLE INFO}

Article history

Received: September 01, 2017

Accepted: October 21, 2017

Published: October 31, 2017

Volume: 5 Issue: 4

Conflicts of interest: None

Funding: None.

\begin{abstract}
Objective: The purpose of this investigation was to determine the differences in collegiate and recreationally trained soccer players in sprint, vertical jump, and balance performance. Methods: Twenty-one soccer players, twelve Division II collegiate and nine recreationally trained volunteered to participate. Session one acted as a familiarization day, where the participants were familiarized with testing day protocols. During testing day, participants performed a dynamic warm-up, followed by balance measurements, three countermovement vertical jumps, and proagility shuttle test. Results: There were no significant ( $p>0.05)$ differences between groups in the all balance variables. Collegiate soccer players had a significantly $(\mathrm{p}<0.05)$ greater peak velocity in vertical jump then recreationally trained soccer players. There were significant differences $(\mathrm{p}<0.05)$ between groups for maximum for split velocities, where collegiate soccer players were greater than recreationally trained soccer players. There were no significant $(p>0.05)$ differences in groups in all other variables. Conclusion: These results indicate that collegiate, Division II, soccer players had greater vertical jumping and sprinting velocities when compared to recreationally trained soccer players. These results may have been impacted by the lack of resistance training background in either of the two groups. With the addition of more time on a collegiate resistance training program, it is very likely the Division II athletes will see a significant increase in all balance, sprint, and vertical jump performance measures compared to recreationally trained players who receive little to no specialized resistance training.
\end{abstract}

Key words: Postural Stability, Sprint Performance, Power Output, Jumping

\section{INTRODUCTION}

Distinguishing athletic performance between levels of competition in sports is essential in determining athlete success (Cometti, Maffiuletti, Pousson, Chatard, \& Maffulli, 2001), establishing normative values (Butler, Southers, Gorman, Kiesel, \& Plisky, 2012; Reilly, Williams, Nevill, \& Franks, 2010), assessing risk for injury (Agel, Evans, Dick, Putukian, \& Marshall, 2007), and designing strength and conditioning programs specific to the needs of its players (Gissis et al., 2006; Kaplan, Erkmen, \& Taskin, 2009; Wisløff, Castagna, Helgerud, Jones, \& Hoff, 2004). Soccer is imperative to investigate due to its high rate of injury (Agel et al., 2007) and unique performance demands. Regardless of competition level, elements necessary for player success include the ability to sprint short distances at high velocities (Cometti et al., 2001; Davis, Brewer, \& Atkin, 1992; Gissis et al., 2006; Kaplan et al., 2009; Reilly et al., 2010; Jason D. Vescovi, 2012)subelite and amateur soccer players to clarify what parameters distinguish the top players from the less successful. We tested 95 soccer players from the French first division (elite, rapidly change direction (Salaj \& Markovic,
2011; D. P. Wong, Chan, \& Smith, 2012), dynamically maintain a stable position to avoid injury during movement(Butler et al., 2012), and produce large amounts of explosive power (Cometti et al., 2001; Jovanovic, Sporis, Omrcen, \& Fiorentini, 2011). These factors should be included in a testing protocol if athletic performance is assessed across levels of play in soccer. Previous research shows sprint performance distinguishes between levels of play in soccer (Cometti et al., 2001; Gissis et al., 2006; Kaplan et al., 2009; Reilly et al., 2010; Jason D. Vescovi, 2012). Although generally recognized as aerobic sport, matches are interspersed with several short (10-30 m) sprints roughly every 90 seconds (Jason D. Vescovi, 2012; Wisløff et al., 2004) which constitutes about $1-11 \%$ of the total distance traveled (Wisløff et al., 2004) in a game. Studies using linear sprint testing protocols have discriminated between elite levels of soccer athletes, where the more advanced level produced significantly faster times (Haugen, Tønnessen, \& Seiler, 2013; Jason D. Vescovi, 2012) and higher velocities (Kalapotharakos et al., 2006). Researchers using similar testing protocols also distinguished elite level soccer athletes from recreationally trained players (Cometti et al., 2001; Gissis 
et al., 2006; Reilly et al., 2010). It's important to include recreationally trained players since the sport is not limited to high level athletes. Desires to decrease injury and increase athletic performance should extend to the amateur level. Although existing literature holds significance, testing protocols have only included linear sprints. In a game situation, soccer players are required to sprint while rapidly changing direction (Salaj \& Markovic, 2011; D. P. Wong et al., 2012). How quickly a player is able to change directions without slowing down is a vital performance variable (Salaj \& Markovic, 2011; D. P. Wong et al., 2012). Designing a protocol to include a pro-agility shuttle test may more accurately simulate game situation sprinting demands. Furthermore, previous research indicates dynamic balance performance to be another classifying variable between levels of competition in soccer (Bressel, Yonker, Kras, \& Heath, 2007; Butler et al., 2012; Davlin, 2004)professional, elite, or Olympic levels, or their individual coaches believed the athlete performed comparably to these levels. High level male and female gymnasts $(\mathrm{n}=57, \mathrm{M}$ age $=17.3 \mathrm{yr} ., \mathrm{SD}=4.1$. Rapidly changing directions while sprinting requires balance and the capacity to dynamically maintain a stable position over a body's center of mass (Butler et al., 2012). Inadequate dynamic balance capability in soccer players has been correlated with a greater risk for injury (Butler et al., 2012). Having a reliable means to evaluate dynamic balance across all classes of experience in soccer is necessary due to an elevated injury risk to the lower extremities.

Researchers have used a number of methods to measure dynamic balance (Bressel et al., 2007; Butler et al., 2012; Davlin, 2004), so the challenge becomes finding a reliable measurement. One system proven in literature to produce reliable values is the Biodex Balance System SD (Parraca et al., 2011). This method has not yet been used to discriminate between levels of play in soccer; however, it has been used in several studies to assess balance and injury risk. Using the Biodex Balance System SD will increase the reliability and decrease the likelihood of errors in measurement. Moreover, vertical jump measures are able to distinguish levels of competition in soccer (Cometti et al., 2001; Wisløff et al., 2004). Explosive lower body power is required while rapidly changing directions during a sprint (Gissis et al., 2006; Wisløff et al., 2004). Previous research examining lower body power in young soccer players indicate the more elite levels having greater countermovement vertical jump heights (VJH) compared to other sub-elite, and recreationally trained players (Gissis et al., 2006). These analyses used the Vertec ${ }^{\circledR}$ to record vertical jump height and made calculated lower body power estimations (Gissis et al., 2006); however, other exact measures of peak power, peak force, peak velocity, and rate of force development can be recorded with the utilization of a force plate.

Due to existing gaps in literature concerning the means of distinguishing athletic performance between levels of play in soccer, the purpose of our study was to investigate the differences in male collegiate and recreationally trained soccer players on balance, sprint, and vertical jump performance measures. Assessments will be made for dynam- ic balance using the Biodex Balance System SD (Balance System $^{\text {TM }}$ SD, Shirley, NY, USA), vertical jump measures using a Vertec $\AA$ (floor model) and force plates (AMTI model BP600900, Watertown, MA, USA), and sprint performance using timing gates (Swift Performance Speedlight Timing Systems, Wacol, Australia) during a pro-agility shuttle test. We hypothesize our results will be consistent with existing literature and predict the collegiate level athletes to perform better across all performance variables in comparison to recreationally trained players.

\section{METHODS}

\section{Participants}

Twenty-one soccer players, twelve Division II collegiate (age $20.33 \pm 1.66 \mathrm{yrs}$; height $178.02 \pm 6.63 \mathrm{~cm}$; weight $74.63 \pm$ $5.73 \mathrm{~kg}$ ) and nine recreationally trained (age 23.22 $\pm 3.41 \mathrm{yrs}$; height $174.32 \pm 5.43 \mathrm{~cm}$; weight $72.14 \pm 11.79 \mathrm{~kg}$ ) volunteered to participate in one familiarization session and one testing session, separated by at least $24 \mathrm{hrs}$ each. Recreationally trained players were required to have at least one year of amateur soccer experience. Division II athletes were recruited from California State University of San Bernardino's collegiate soccer team. An informed consent describing the testing protocol and a Physical Activity Readiness Questionnaire (PARQ) was completed on familiarization day. Participants were healthy and free of any lower body musculoskeletal or orthopedic injuries. The protocol was verbally described and anthropometrics (age, height, and weight) were recorded.

\section{Protocol}

Following the completion of an informed consent, PARQ, and collection of anthropometrics on familiarization day, a dynamic warm-up was performed including: two sets of a $15 \mathrm{~m}$ jog, exaggerated lunges, high knees, walking planks, and leg swings. Next, participants were familiarized with balance, vertical jump, and sprint protocols. Balance tests were completed on the Biodex Balance System SD and consisted of three practice trials each of Static Balance (SB), Limits of Stability (LOS), and Single Leg Balance (SLB) tests. Next, three practice trials of a countermovement vertical jump (CMJ) were performed using a Vertec $\AA$ and force plate. Lastly, three practice trials of a pro-agility sprint protocol were completed with a 3-minute rest between each trial. Participants returned to the laboratory at least 24 hrs following familiarization for testing session.

\section{Biodex Balance System SD}

$\mathrm{SB}$, LOS, and SLB tests were completed on the BioDex Balance System SD. The SB and the SLB test consisted of three successful trials at 20 s each with a 10 s rest between each trial. The LOS test consisted of three trials until completion of task, with a $10 \mathrm{~s}$ rest between each trial. A trial was deemed successful if the participant did not touch the side hand rails and kept only one leg on the platform for single leg proto- 
cols. The level of each test was determined using a very unstable to static spectrum. Level one is the most difficult and allows for the maximum degrees of variation from horizontal in platform movement and there is no platform movement in the static level. SB was set to static, LOS to level 6, and SLB to level 4. SLB measured Overall, Anterior/Posterior, and Medial/Lateral degrees from horizontal. The LOS test measured Overall, Forward, Backward, Right, Left, Forward/Right, Forward/Left, Backward/Right, Backward/Left degrees from horizontal as well as time to completion. Since there is no movement in the platform, SB outcome variables measure anterior, posterior, right, and left sway from a center point (Oliver \& Di Brezzo, 2009).

\section{Vertec and Force Plate}

Three successful CMJ trials were performed with $15 \mathrm{~s}$ rest between each jump (Dabbs, Brown, \& Garner, 2014). Participants were instructed to jump as high and as quickly as possible using a countermovement arm swing during each jump. A Vertec ${ }^{\circledR}$ was used to measure VJH and an AMTI force plate was used to measure peak force $(\mathrm{PF})$, peak power output (PPO), peak velocity (PV), and rate of velocity development (RVD). In between each jump, the force plate was zeroed out to ensure accurate measurements.

\section{Timing Gates}

Participants performed three successful trials of a pro-agility shuttle test requiring a change in direction twice during the sprint (Vescovi, Brown, \& Murray, 2006). The protocol included a 20yd sprint with four, 5yd split times. Swift Performance Speed Light laser timing gates were used to record exact split and total times. Participants were instructed to stand as close to the starting point as possible marked by a taped line and go on the sound of a beep set off by the first set of timing gates. Minimum reaction time (RT), minimum split times (ST), minimum total times (TT), maximum split time velocities (STV), and maximum total velocity (TV) were calculated.

\section{Statistical Analysis}

To analyze differences between the recreational and collegiate soccer players, independent t-tests were conducted for each variable in SB, LOS, and SLB balance measures. RT, ST, TT, and STV were calculated from the pro-agility test. The Vertec $\AA$ was used to measure VJH and the AMTI force plate measured vertical forces and calculated PPO, PV, PF, and RVD.

\section{RESULTS}

There was a significant $(\mathrm{p}=.049)$ difference between groups with collegiate athletes $(3.05 \pm .27 \mathrm{~m} / \mathrm{s})$ being greater than recreationally trained $(2.8 \pm .25 \mathrm{~m} / \mathrm{s})$ soccer players for PV during the vertical jump (Figure 1). There were no significant $(p>0.05)$ differences between groups in all other vertical jump measures. There were no significant $(p>0.05)$ differences in balance measures between groups in all balance measures. There were significant differences $(p=0.02)$ between groups for sprint TV, where collegiate soccer players $(3.85 \pm .13 \mathrm{~m} / \mathrm{s})$ were greater than recreationally trained $(3.69 \pm .16 \mathrm{~m} / \mathrm{s})$ soccer players. There were significant differences $(p=0.03)$ between groups for sprint maximum STV one, STV two, and STV four where collegiate soccer players $(3.80 \pm .2 \mathrm{~m} / \mathrm{s})$ were greater than recreationally trained $(3.11 \pm .1 .0 \mathrm{~m} / \mathrm{s})$ soccer players. There were no significant $(p>0.05)$ differences between groups for in all other sprint measures (Table 1).

\section{DISCUSSION}

The objective of our study was to examine the differences in male collegiate and recreationally trained soccer players on balance, sprint, and vertical jump performance measures. Significant differences were found between levels of play for peak velocity during a vertical jump, maximum total sprint velocity, and maximum sprint velocity during time split one. Significant differences were not found between levels of play for all other balance, sprint, and vertical jump measures.

Previous research examining lower body power in young soccer players indicated more elite levels having greater CMJ height compared to other sub-elite, and recreationally trained players (Gissis 2006); however, studies recording force and velocity vertical jump measures using similar soccer populations could not be found. The current investigation measured peak power, peak velocity, and peak force variables while performing a CMJ and found differences between the two groups was higher in collegiate athletes for peak velocity. Power is the product of force times velocity, where increases in both force and velocity result in an increase in power production capability (Cormie, McBride, \& McCaulley, 2009). Although the DII soccer athletes were able to produce higher velocities and move more quickly, the lack of significant difference in peak force and peak power may be explained by their inability to generate enough force to change the power output. Other subsequent research determined dynamic balance performance to be a classifying

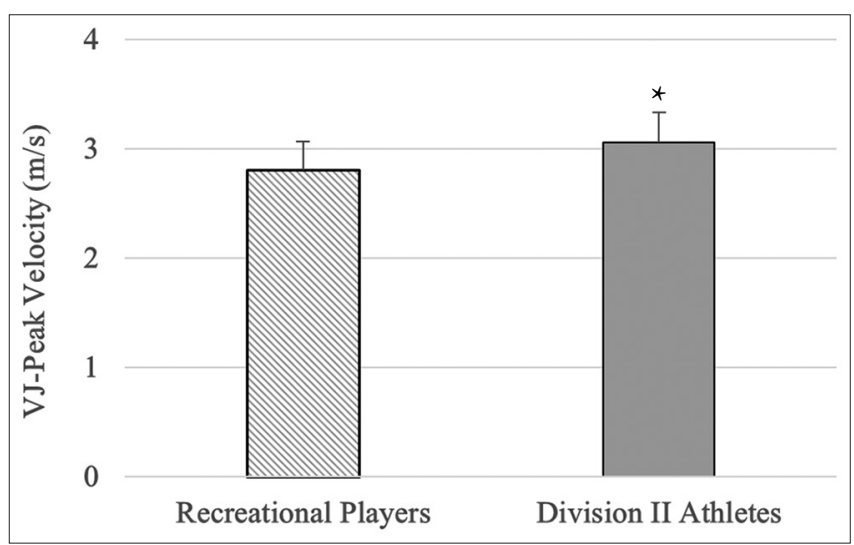

Figure 1: Means \pm Standard Deviations for Division II soccer athletes and Recreationally Trained soccer players, indicating a significantly $(* \mathrm{p}<0.05)$ greater Peak Velocity during a vertical jump. 
Table 1. Differences between recreational players and Division II Athletes in balance, vertical jump, and sprint performance measures

\begin{tabular}{|c|c|c|c|c|}
\hline \multirow[t]{2}{*}{ Measure } & \multicolumn{2}{|c|}{ Recreational players } & \multicolumn{2}{|c|}{ Division II Athletes } \\
\hline & Mean & SD & Mean & SD \\
\hline \multicolumn{5}{|l|}{ SB } \\
\hline Overall & 0.69 & 0.68 & 0.59 & 0.56 \\
\hline $\mathrm{A} / \mathrm{P}$ & 0.54 & 0.7 & 0.48 & 0.47 \\
\hline $\mathrm{M} / \mathrm{L}$ & 0.33 & 0.38 & 0.24 & 0.26 \\
\hline \multicolumn{5}{|l|}{ SLB right } \\
\hline Overall & 2.06 & 1.22 & 1.86 & 0.7 \\
\hline $\mathrm{A} / \mathrm{P}$ & 1.34 & 0.66 & 1.33 & 0.56 \\
\hline $\mathrm{M} / \mathrm{L}$ & 1.46 & 1.1 & 1.13 & 0.48 \\
\hline \multicolumn{5}{|l|}{ SLB left } \\
\hline Overall & 2.04 & 0.91 & 1.71 & 0.52 \\
\hline $\mathrm{A} / \mathrm{P}$ & 1.44 & 0.63 & 1.24 & 0.33 \\
\hline $\mathrm{M} / \mathrm{L}$ & 1.23 & 0.62 & 1 & 0.44 \\
\hline \multicolumn{5}{|l|}{ LOS } \\
\hline Overall & 27.56 & 9.34 & 25.75 & 6.81 \\
\hline Forward & 36.67 & 15.43 & 40 & 10.94 \\
\hline Backward & 43.56 & 13.24 & 46.08 & 15.28 \\
\hline Right & 31.56 & 14.06 & 27.33 & 10.19 \\
\hline Left & 32.22 & 13.05 & 26.5 & 9.43 \\
\hline $\mathrm{F} / \mathrm{R}$ & 31.89 & 11.47 & 29.5 & 10.06 \\
\hline $\mathrm{F} / \mathrm{L}$ & 28.67 & 13.24 & 31.08 & 8.31 \\
\hline $\mathrm{B} / \mathrm{R}$ & 35.22 & 12.34 & 30 & 9.21 \\
\hline $\mathrm{B} / \mathrm{L}$ & 34.78 & 8.54 & 34.58 & 9.42 \\
\hline VJH (cm) & 54.61 & 7.4 & 60.21 & 8.65 \\
\hline PPO (W) & 4527.79 & 516.69 & 4981.3 & 670.92 \\
\hline $\mathrm{PF}(\mathrm{N})$ & 1807.57 & 304.81 & 1984.95 & 376.2 \\
\hline $\operatorname{RVD}\left(\mathrm{m} / \mathrm{s}^{2}\right)$ & 7685.73 & 2712.91 & 8711 & 3888.96 \\
\hline $\operatorname{Max}$ TV $(\mathrm{m} / \mathrm{s})$ & $3.69 *$ & 0.16 & 3.85 & 0.13 \\
\hline Max STV 1 (m/s) & $3.12 *$ & 1.01 & 3.81 & 0.2 \\
\hline Max STV 2 (m/s) & $2.97 *$ & 1.04 & 3.65 & 0.26 \\
\hline Max STV 3 (m/s) & 4.06 & 1.75 & 4.86 & 0.38 \\
\hline Max STV 4 (m/s) & $2.92 *$ & 0.87 & 3.6 & 0.29 \\
\hline Min ST 1 & $1.29 *$ & 0.11 & 1.19 & 0.07 \\
\hline Min ST 2 & 1.19 & 0.11 & 1.21 & 0.09 \\
\hline Min ST 3 & 0.94 & 0.07 & 0.92 & 0.06 \\
\hline Min ST 4 & 1.33 & 0.12 & 1.22 & 0.16 \\
\hline Min RT & 0.49 & 0.13 & 0.48 & 0.11 \\
\hline Min TT & 4.76 & 0.3 & 4.54 & 0.22 \\
\hline
\end{tabular}

$\mathrm{SB}=$ static balance; $\mathrm{SLB}=$ single leg balance; $\mathrm{A} / \mathrm{P}=$ anterior/posterior; $\mathrm{M} / \mathrm{L}=$ medial/lateral; $\mathrm{LOS}=$ limits of stability; $\mathrm{F} / \mathrm{R}=\mathrm{Forward} /$ Right; $\mathrm{F} / \mathrm{L}=$ =forward/left; $\mathrm{B} / \mathrm{R}=$ backward/right; $\mathrm{B} / \mathrm{L}=$ =backward/left; $\mathrm{VJH}=$ =ertical jump height; $\mathrm{PV}=$ peak velocity; $\mathrm{PPO}=$ peak power output; $\mathrm{PF}=$ peak force; $\mathrm{RVD}=$ rate of velocity development; $\mathrm{STV}=$ split time velocity; $\mathrm{ST}=$ split time; RT=reaction time. * Indicates a significant difference, where Division II Soccer Athletes had a greater PV, Max TV, Max STV 1, Max STV 2, and Max STV 4 than Recreationally Trained Soccer Players

variable between levels of competition in soccer (Bressel et al., 2007; Butler et al., 2012; Davlin, 2004) yet the current investigation concluded no significant differences between the two groups in all balance measures. A previous study also indicate balance performance and force production measures to simultaneously increase following resistance training programs (Manolopoulos et al., 2016). Our lack of differences found between levels may be due to a relatively 
new university strength and conditioning program prior to data collection for the Division II athletes, which may have resulted in a decrease in performance, specifically balance and force production.

Levels of play in soccer have also been distinguished through linear sprint performance (Cometti et al., 2001; Gissis et al., 2006; Kaplan et al., 2009; Reilly et al., 2010; Jason D. Vescovi, 2012); however, similar research utilizing change in direction protocols did not exist which may more closely simulate game situation demands. Our study's methodology included a pro-agility test resulting in collegiate level soccer athletes having significantly higher maximum and split-one velocities in comparison to the recreationally trained players. Although it may have been too early in the newly implemented strength and conditioning program to attribute resistance training to higher velocity measures in the Division II athletes, the measures may be accredited to a more extensive time period of soccer experience and pure exposure to a higher level of competition. Once again, the study's results may have been partially impacted by the lack of resistance training background in either of the two groups. With the addition of a longer time spent resistance training, it is very likely the Division II athletes will see a significant increase in all balance, sprint, and vertical jump performance measures compared to recreationally trained players who receive little to no specialized resistance training. If improvements are shown, this further validates the performance benefits and need of a quality resistance training program for soccer players (Grieco, Cortes, Greska, Lucci, \& Onate, 2012; Negra, Chaabene, Hammami, Hachana, \& Granacher, 2016; P. Wong, Chaouachi, Chamari, Dellal, \& Wisloff, 2010).

Some limitations in the current investigation may be the differences between levels of the soccer players, for instance if the recreationally trained athletes were compared to Division I or professional level team, results may have been different. Additionally, testing the Division II athletes further along in their new strength and conditioning program would have been more representative of their overall performance. Although our study observed differences in velocity measures between Division II collegiate soccer athletes and recreationally trained participants, more research is needed evaluating performance measures in all levels of play. This would contribute to improving resources at hand for professionals working with soccer athletes, so they can properly make assessments of injury risks, normative values, and athlete performance across all divisions of competition. Future research would include investigating Division II soccer players following more routine resistance training and agility training when compare to recreationally trained players. Additionally, investigating a variety of sports comparing highly trained athletes and recreationally trained players.

\section{CONCLUSION}

In conclusion, it appears that there is a distinct difference between Division II and recreationally trained soccer athletes in vertical jump and sprint performance. However, training level seems to have no effect on balance performance in the current investigation. These results may have been impacted by the lack of resistance training background in either of the two groups. With the addition of more time on a collegiate resistance training program, it is very likely the Division II athletes will see a significant increase in all balance, sprint, and vertical jump performance measures compared to recreationally trained players who receive little to no specialized resistance training.

\section{REFERENCES}

Agel, J., Evans, T. A., Dick, R., Putukian, M., \& Marshall, S. W. (2007). Descriptive Epidemiology of Collegiate Men's Soccer Injuries: National Collegiate Athletic Association Injury Surveillance System, 19881989 Through 2002-2003. Journal of Athletic Training, 42(2), 270.

Bressel, E., Yonker, J. C., Kras, J., \& Heath, E. M. (2007). Comparison of Static and Dynamic balance in female and collegiate soccer, basketball, and gymnastics. Journal of Athletic Training, 42, 42-46.

Butler, R. J., Southers, C., Gorman, P. P., Kiesel, K. B., \& Plisky, P. J. (2012). Differences in Soccer Players' Dynamic Balance Across Levels of Competition. Journal of Athletic Training, 47(6), 616.

Cometti, G., Maffiuletti, N. A., Pousson, M., Chatard, J. C., \& Maffulli, N. (2001). Isokinetic strength and anaerobic power of elite, subelite and amateur French soccer players. International Journal of Sports Medicine, 22(1), 45-51.

Cormie, P., McBride, J. M., \& McCaulley, G. O. (2009). Power-time, force-time, and velocity-time curve analysis of the countermovement jump: impact of training. Journal of Strength and Conditioning Research, 23(1), 177-186.

Dabbs, N. C., Brown, L. E., \& Garner, J. C. (2014). Effects of Whole Body Vibration on Vertical Jump Performance Following Exercise Induced Muscle Damage. International Journal of Kinesiology and Sports Science, 2(1), 24-30.

Davis, J. A., Brewer, J., \& Atkin, D. (1992). Pre-season physiological characteristics of English first and second division soccer players. Journal of Sports Sciences, $10(6), 541-547$.

Davlin, C. D. (2004). Dynamic Balance in High Level Athletes. Perceptual and Motor Skills, 98(3_suppl), 1171-1176.

Gissis, I., Papadopoulos, C., Kalapotharakos, V. I., Sotiropoulos, A., Komsis, G., \& Manolopoulos, E. (2006). Strength and speed characteristics of elite, subelite, and recreational young soccer players. Research in Sports Medicine, 14(3), 205-214.

Grieco, C. R., Cortes, N., Greska, E. K., Lucci, S., \& Onate, J. A. (2012). Effects of a Combined Resistance-Plyometric Training Program on Muscular Strength, Running Economy, and VO2 Peak in Division I Female Soccer Players. Journal of Strength Conditioning Research, 26(9), 2570-2576.

Haugen, T. A., Tønnessen, E., \& Seiler, S. (2013). Anaerobic performance testing of professional soccer players 
1995-2010. International Journal of Sports Physiology and Performance, 8(2), 148-156.

Jovanovic, M., Sporis, G., Omrcen, D., \& Fiorentini, F. (2011). Effects of speed, agility, quickness training method on power performance in elite soccer players. Journal of Strength and Conditioning Research, 25(5), 1285-1292.

Kalapotharakos, V. I., Strimpakos, N., Vithoulka, I., Karvounidis, C., Diamantopoulos, K., \& Kapreli, E. (2006). Physiological characteristics of elite professional soccer teams of different ranking. The Journal of Sports Medicine and Physical Fitness, 46(4), 515-519.

Kaplan, T., Erkmen, N., \& Taskin, H. (2009). The evaluation of the running speed and agility performance in professional and amateur soccer players. Journal of Strength and Conditioning Research, 23(3), 774-778.

Manolopoulos, K., Gissis, I., Galazoulas, C., Manolopoulos, E., Patikas, D., Gollhofer, A., \& Kotzamanidis, C. (2016). Effect of Combined Sensorimotor-Resistance Training on Strength, Balance, and Jumping Performance of Soccer Players. Journal of Strength and Conditioning Research, 30(1), 53-59.

Negra, Y., Chaabene, H., Hammami, M., Hachana, Y., \& Granacher, U. (2016). Effects of High-Velocity Resistance Training on Athletic Performance in Prepuberal Male Soccer Athletes. Journal of Strength and Conditioning Research, 30(12), 3290-3297.

Oliver, G. D., \& Di Brezzo, R. (2009). Functional balance training in collegiate women athletes. Journal of Strength and Conditioning Research, 23(7), 2124-9.

Parraca, J. A., Sánchez-Toledo, O., Rufino, P., Carbonell Baeza, A., García-Molina, A., A, V., Gusi Fuertes, N. (2011). Test-Retest reliability of Biodex Balance SD on physically active old people. Journal of Human Sport and Exercise, 6(2), 444-450.

Reilly, T., Williams, A. M., Nevill, A., \& Franks, A. (2010). A multidisciplinary approach to talent identification in soccer. Journal of Sports Sciences. 18(9), 695-702.

Salaj, S., \& Markovic, G. (2011). Specificity of Jumping, Sprinting, and Quick Change-of-Direction Motor Abilities. Journal of Strength and Conditioning Research, 25(5), 1249-1255.

Vescovi, J. D., Brown, T. D., \& Murray, T. M. (2006). Positional Characteristics of physical performance in Division I college female soccer players. Journal of Sports Medicine and Physical Fitness, 46, 221-226.

Vescovi, J. D. (2012). Sprint speed characteristics of high-level American female soccer players: Female Athletes in Motion (FAiM) Study. Journal of Science and Medicine in Sport, 15(5), 474-478.

Wisløff, U., Castagna, C., Helgerud, J., Jones, R., \& Hoff, J. (2004). Strong correlation of maximal squat strength with sprint performance and vertical jump height in elite soccer players. British Journal of Sports Medicine, 38(3), 285-288.

Wong, D. P., Chan, G. S., \& Smith, A. W. (2012). Repeated-Sprint and Change-of-Direction Abilities in Physically Active Individuals and Soccer Players: Training and Testing Implications. Journal of Strength and Conditioning Research, 26(9), 2324-2330.

Wong, P., Chaouachi, A., Chamari, K., Dellal, A., \& Wisloff, U. (2010). Effect of Preseason Concurrent Muscular Strength and High-Intensity Interval Training in Professional Soccer Players. Journal of Strength and Conditioning Research, 24(3), 653-660. 